\title{
MAGNETIC RESONANCE SPECTROSCOPY OF NORMAL APPEARING WHITE MATTER IN EARLY RELAPSING-REMITTING MULTIPLE SCLEROSIS
}

\author{
Enas El Sayed Mohamed Ahmed*, Mahmoud Ashraf Zaiton*, HananAbd ElAzim Hassan*, Wael \\ Mahmoud ElSayed* , Khalid Mohamed Shawki** \\ Neurology * and Radiolgy** departments faculty of Medicine; zagazig university.
}

Back ground: Multiple sclerosis (MS) is an inflammatory disease in which the insulating covers of nerve cells in the brain and spinal cord are damaged .MS is a chronic disease of the central nervous system characterised by multicentral inflammation and myelin destruction .

Magnetic resonance imaging (MRI) is now widely used for diagnosing MS and detecting clinically silent lesions. However, correlations found between conventional techniques, such as T2-weighted lesion load, and disability, are weak or absent and therefore, neither the magnetic resonance techniques nor clinical measurements are gold standard to assess disease or disability.Alteration of NAWM is of great importance because its true patho-physiological significance is not completely understood. Decrease in N-Acetyl Aspartate (NAA) has been used as an marker of axonal damage or loss that presumably appears secondary to inflammation or demyelination ,although primary axonal damage is not excluded .

Objectives : Demonstrate the limitation of convenential magnetic resonance techniques.

Observation the pathological processes in NAWM by MRS.

Correlation between the results of MRS and the physical disability of the patients using EDSS .

Subjects and methods: This study was carried out on 23 patients with clinically definite multiple sclerosis patients, who met the criteria of clinically definite MS according to McDonald criteria 2010, selected from Neurology Department, Zagazig University Hospitals [10].

Results: We found that the mean value of NAA and the mean ratios of NAA/CR and NAA/ CHO were significant lower in the Cases but the mean values of MI and GLX are significant higher. . Also there was significant negative correlation between the mean value of NAA in MS plaque by MRS and EDSS score as a measure of disability.

There was significant negative correlation between the mean value of NAA in NAWM and EDSS score .

Discussion: We have clearly demonstrated a correlation between an MRS measure and EDSS. EDSS seems to reflect the existence of irreversible disability probably related to axonal degeneration.

Key wards

Multiple sclerosis; Magnetic resonance imaging;Normal appearing white mater;Proton magnetic resonance spectroscopy;Expanded Disability Status Scale

Corresponding author: Enas El Sayed Mohamed Ahmed Mobile:01001200386

Email:dr_enasneuro@yahoo.com

\section{INTRODUCTION}

$\mathbf{M}$ ultiple sclerosis (MS) is an immunemediated inflammatory disease that attacks myelinated axons in the central nervous system (CNS), destroying the myelin and the axon in variable degrees. In most cases, the disease follows a relapsing-remitting pattern, with short-term episodes of neurologic deficits that resolve completely or almost completely. A minority of patients experience steadily progressive neurologic deterioration [1].

\section{MS distribution}

It is recognised that MS is unevenly but nonrandomly distributed throughout the world and that environmental factors play a significant role in the onset of MS. Many epidemiology studies have been performed to investigate these phenomena. Several of these studies support the existence of a gradient of MS 
prevalence, which increases with distance from the equator in both northern and southern hemispheres ${ }^{[2]}$.

\section{Etiology}

The cause of MS is unknown, but it is likely that multiple factors act in concert to trigger the disease. It has been hypothesized that MS results when an environmental agent or event acts in concert with a genetic predisposition to immune dysfunction ${ }^{[3]}$.

\section{Axonal Damage in Multiple Sclerosis}

Historically, axonal damage has been recognized as a histopathological hallmark of MS since the very early descriptions of the disease by Charcot in the late $1800 \mathrm{~s}^{[4}$ ]. More recent clinical, histopathological, and neuroimaging evidence has shed new light on these early findings and supported the concept that the debilitating disease course and longterm disability in MS patients was consequent to axonal loss possibly consequent to demyelination $\left.{ }^{[5}\right]$.The features of axonal damage in MS, however, were similar to those detected in other neurological diseases lacking demyelination, such as amyotrophic lateral sclerosis (ALS), and suggested the possibility that axonal damage in MS might be concurrent to demyelination, but not necessarily consequent to myelin destruction ${ }^{[6,7]}$.

\section{Clinical Rating Scales}

A patient may be rated according to several clinical disability scales, on the basis of findings on the history and physical examination. The most widely accepted of these is the 10-point Kurtzke Expanded Disability Status Scale (EDSS), which was developed originally in 1955 as the Disability Status Scale and has been revised over the years [8].

The EDSS assigns a severity score to the patient's clinical status that ranges from $0-10$ in increments of 0.5. The scores from grades 0-4 are determined using functional systems (FS) scales that evaluate dysfunction in the following 8 neurologic systems:

- Pyramidal,Cerebellar ,Brainstem,Sensory,Bladder and bowel, Vision, Cerebral, Other
Advantages of the EDSS are that it is widely used clinically, is easy to administer, and requires no special equipment.

Proton magnetic resonance (MR) imaging plays an essential role in the management of patients with a wide range of neurological conditions. While conventional MR imaging provides important structural information, data on underlying brain function is often limited. The generation of a spectrum of brain metabolites by MR spectroscopy provides the clinician with information on the regional chemical environment ${ }^{[9]}$.

\section{Proton MR Spectroscopy}

Over the years, scientists have obtained MR spectra using a variety of nuclei ${ }^{[10]}$.However, the high sensitivity of the hydrogen $\left({ }^{1} \mathrm{H}\right)$ nucleus, its abundance within certain neurometabolites and the fact that the technique can be performed using standard clinical MR imaging machines makes hydrogen the principal nucleus applicable to spectroscopic investigation associated with clinical imaging of the brain ${ }^{[11]}$.

The proton MR spectrum comprises a set of resonances (peaks) distributed along the $\mathrm{X}$-axis, labelled in parts per million (ppm). The amplitude of the resonances is measured on the y-axis typically using an arbitrary scale. Three prominent peaks are consistently seen: $\mathrm{N}$-acetyl aspartate (NAA) at 2.02; Creatine $(\mathrm{Cr})$ at 3.02; and Choline (Cho) at 3.2 .Although the positions of the resonances along the $\mathrm{x}$-axis are constant, the relative heights of the resonances can differ depending on various MR imaging parameters ${ }^{[12]}$.

\section{SUBJECTS AND METHODS}

This study was carried out on 23 patients with clinically definite multiple sclerosis patients, who met the criteria of clinically definite MS according to McDonald criteria 2010, selected from Neurology Department, Zagazig University Hospitals ${ }^{[13]}$.

Ten healthy volunteers with similar age and gender distribution as control group were included in this study. The study was approved by the Ethical Committee of our Faculty and 
informed written consent was obtained from patients and controls.

Patients were classified according to clinical course as having recurrent relapse with partial remission and no progression between attacks (relapsing remitting course)

The patients group:

This group included 23 Patients with clinically definite relapsing remitting MS according to the Mc Donald criteria ${ }^{[14]}$. Their ages ranged from 17 year to 45 year, with disease duration ranged from 1 to 12 years

\section{The control group:}

Ten apparently healthy unmedicated subjects, of matched age and gender served as a control group.

\section{All patients were subjected to the following: (1) History and examination:}

- $\quad$ Detailed history taking about age of onset, duration of disease, number of relapse and current treatment(disease modifying therapy).

- $\quad$ The duration of the disease (measured in years). Patient's age at first symptoms ..

- Assessment of disease severity by the Expanded Disability Status Scale (EDSS) ${ }^{[15]}$.

\section{(2)Routine laboratory investigations:}

(3) Radiological evaluation was performed in Zagazig University Radiology MRI Unit: MR images were acquired using a 1.5T Philips using a standard quadrature head coil.
Prior to H-MRS examination each patient's disability was measured using EDSS . The H-MRS was performed the same day as the neurological examination by the same radiologist at the same center using the same MRI scanner and following the same protocol. The radiologist was blinded to the neurological examination results and all the neurologists were blinded to the MRS results.

At the beginning of the study MRI and H-MRS were also performed on 10 healthy, age matched control subjects with not known systemic or neurological disease by the same radiologist using the same MRI scanner and following the same protocol as well.

These conventional MR images were used to position a spectroscopic volume of interest (VOI) in one or two areas with demyelinating lesions and one area of normal appearing white matter in MS patients and in one area of normal white matter in the healthy control group The number of peaks fitted included the chemical shift ranges:

- $\quad N$-acetylasparate (NAA) at $2.0 \mathrm{ppm}$.

$\circ \quad$ Creatine/phosphocreatine $(\mathrm{Cr})$ at $3.0 \mathrm{ppm}$.

○ Choline compounds (Cho) at $3.2 \mathrm{ppm}$.

○ Myo-inositol (mI) at $3.5 \mathrm{ppm}$.

- Glutamine-glutamate-GABA complex

(Glx) between 2.1 and 2.5 ppm 


\section{RESULTS}

Table (1) : Comparison between the different metabolic values of MS plaques and that of NAWM of the patient group by MRS .

\section{Patient group $\quad$ p}

\begin{tabular}{|c|c|c|c|}
\hline & $\begin{array}{l}\text { MS plaques } \\
\text { Mean } \pm \text { SD }\end{array}$ & $\begin{array}{l}\text { NAWM } \\
\quad \text { Mean } \pm \text { SD }\end{array}$ & \\
\hline NAA & $1.9860 \pm 0.172$ & $1.968 \pm 0.2048$ & 0.891 \\
\hline$\overline{C R}$ & $3.0266 \pm 0.0118$ & $3.0278 \pm 0.01389$ & 0.270 \\
\hline CHO & $3.2155 \pm 0.0135$ & $3.2160 \pm 0.012$ & 0.376 \\
\hline$\overline{M I}$ & $3.9488 \pm 0.247$ & $4.1053 \pm 0.01475$ & $0.005 *$ \\
\hline GLX & $3.6874 \pm 0.0654$ & $3.7670 \pm 0.04318$ & 0.256 \\
\hline NAA/CR & $1.4815 \pm 1.0438$ & $1.1539 \pm 1.08437$ & 0.112 \\
\hline NAA/CHO & $1.1582 \pm 0.7565$ & $0.9070 \pm 0.77619$ & 0.162 \\
\hline
\end{tabular}

This table shows that the mean value of MI was significant higher in the NAWM than in the MS plaque in the same patient.

Table (2): Comparison between the different metabolic values of NAWM of the patient group and that of the control group by MRS .

\begin{tabular}{llll}
\hline & $\begin{array}{l}\text { Patient group } \\
\text { NAWM } \\
(\mathrm{N}=23)\end{array}$ & $\begin{array}{l}\text { Control group } \\
(\mathrm{N}=10)\end{array}$ & $\mathrm{p}$ \\
\hline $\mathrm{NAA}$ & $1.968 \pm 0.2048$ & $2.0275 \pm 0.00288$ & $0.003^{*}$ \\
\hline $\mathrm{CR}$ & $3.0278 \pm 0.01389$ & $3.0237 \pm 0.00356$ & 0.366 \\
\hline $\mathrm{CHO}$ & $3.2152 \pm 0.1437$ & $3.2160 \pm 0.012$ & 0.881 \\
\hline $\mathrm{MI}$ & $4.1053 \pm 0.01475$ & $3.5887 \pm 0.09979$ & $0.00^{* *}$ \\
\hline GLX & $3.7670 \pm 0.04318$ & $3.6343 \pm 0.08474$ & $0.005^{*}$ \\
\hline NAA/CR & $1.1539 \pm 1.08437$ & $2.235 \pm 0.30725$ & $0.0008^{*}$ \\
\hline NAA/CHO & $0.9070 \pm 0.77619$ & $1.7360 \pm 0.17335$ & $0.001^{*}$ \\
\hline $\begin{array}{l}\text { This table shows that the mean value of NAA } \\
\text { were significant lower in the Cases than the control } \text { (p<0.005) but the mean values of MI and GLX are } \\
\text { significant higher than the control group. }\end{array}$ &
\end{tabular}


Table (3): Correlation between EDSS and clinical data of the patients.

Clinical data of the patients EDSS

\begin{tabular}{lll}
\hline & $\mathbf{r}$ & $\mathrm{p}$ \\
\hline Age & 0.200 & 0.361 \\
\hline Age of onset & 0.074 & 0.738 \\
& & 0.048 \\
\hline Disease duration & $0.416^{*}$ & \\
\hline No of relapse & & 0.001 \\
\hline
\end{tabular}

This table shows that there was significant positive correlation between the duration of the disease and EDSS score as a measure of disability and number of ralapses and EDSS score .

Table (4): Correlation between EDSS and metabolic values of MS Plaques in MRS.

\begin{tabular}{|c|c|c|}
\hline \multirow[t]{2}{*}{ Metabolic values } & \multicolumn{2}{|r|}{ EDSS } \\
\hline & $\mathrm{r}$ & $\mathbf{p}$ \\
\hline NAA & $-0.613 * *$ & 0.002 \\
\hline $\mathbf{C R}$ & -0.307 & 0.154 \\
\hline CHO & -0.277 & 0.201 \\
\hline MI & -0.051 & 0.818 \\
\hline GLX & -0.121 & 0.581 \\
\hline NAA/CR & 0.50 & 0.820 \\
\hline NAA/CHO & -0.366 & 0.086 \\
\hline
\end{tabular}


Table (5): Correlation between EDSS score and the metabolic values of NAWM in MRS. EDSS

$\mathbf{r}$

\begin{tabular}{|c|c|c|}
\hline NAA & - 0.764** & 0.454 \\
\hline $\mathbf{C R}$ & -0.347 & 0.104 \\
\hline CHO & -0.283 & 0.190 \\
\hline MI & -0.295 & 0.172 \\
\hline GLX & -0.231 & 0.288 \\
\hline NAA/CR & $-.013^{*}$ & 0.953 \\
\hline NAA/CHO & -0.179 & 0.419 \\
\hline
\end{tabular}

\section{DISCUSSION}

The major goal of this study was to investigate specific metabolite changes, particularly NAA alterations, in the different areas of NAWM of MS patients using MRS technique and to conduct an analysis of spectroscopy markers of axonal integrity, astrogliosis, and demyelination in vivo and correlation with the clinical disability of the patients using EDSS score. Our results showed the metabolite values and ratios of MS patients (in NAWM and in WML) determined by H-MRS. MS patients in this study from the early stages of the disease, showed higher values for MI in NAWM compared with the MS plaques. These results coincide with others published earlier, using similar techniques ${ }^{[\mathbf{1 6}, 17,18,19,20]}$.

In contrast to our results, Fernando et al ,2004 ${ }^{[21]}$ reported higher absolute concentrations of NAA in NAWM and no significant decreased NAA level in patients with definite MS. The $\mathrm{Cr}$ and Cho in this study were increased in RRMS patients in plaques and NAWM but there was not statistically significant

when compared to the controls. On contrary,other authors found in their study significant elevation in $\mathrm{Cr}$ in RRMS group compared to controls ${ }^{[22]}$.Other studies demonstrated increased Cho levels in MS plaques and NAWM in RRMS, as well as decreased NAA concentrations [24], presenting inflammatory changes and significant axonal damage.

Metabolic ratios are less sensitive than absolute concentrations of individual metabolites ${ }^{[23]}$. When comparing NAA/Cho and NAA/Cr ratios in the lesions, NAWM in patients versus controls, they were clearly reduced, consistent with results obtained in previous studies. The observationis that the magnitude of the reduction varies with the type and clinical form of the disease. Since it was first described, MS has been considered a demyelinating disease of the central nervous system, and until recently axonal loss had not been studied in depth. The $\mathrm{N}$-Acetyl Aspartate levels detected by H-MRS could provide a precise marker of axonal loss [25].

Although the function of myoinositol (mI) is uncertain, we know that it is involved in the polyphosphoinositol second messenger cascade and that it is relatively concentrated in glial 
cells when compared with neurones as was shown by multinuclear spectroscopy techniques performed in rats. So an increase in $\mathrm{mI}$ may reflect neuroglial homeostasis and gliosis . This finding is in agreement with other study that showed significant increase in NAWM Mi. the results provide evidence that $\mathrm{M} I$ is increased in NAWM in early clinical stage of multiple sclerosis ${ }^{[26]}$.

Another observation in this work was significant elevation of glutamate in NAWM when compared with the control group .This was in agreement with the findings of Anders et al $\mathbf{2 0 1 3}^{[27]}$, who also found increased Glu in both acute lesions and in NAWM of MS patients, indicating that elevated levels of Glu are involved in the progression of MS even in this non-lesional MS subtype. Therefore, there is a potential for Glu to be used as a marker of the severity of MS even in patients where no white matter lesions are found ${ }^{[28]}$.

In our study we correlated the EDSS score as a measuere of the disability with the clinical data of all patients, we found significant positive correlation between the number of relapse and the score. These findings coincide with Minneboo et al, 2004$)^{[29]}$, who emphasized the role of number of relapse in long-term prognosis for patients. Moreover, investigations in the past few decades have revealed that many clinical are predictive of long-term outcomes, including sex, higher age and pyramidal symptoms at disease onset, shorter time to second clinical attack, and number of relapses in the early disease phase ${ }^{[31}$ ].

In our study, we studied the clinical disability of our patient, using EDSS score; also we analyzed the metabolic levels of MS plaques and NAWM of $23 \mathrm{MS}$ patients and 10 controls. In the current study, we found a significant correlation between NAA and clinical score using EDSS score in accordance with findings reported by Adalsteinsson et al , 2003 ${ }^{[30]}$. MRS seems to be a potential surrogate marker of disease progression. In contrast to our findings, recently, Rigotti et al 2011 ${ }^{[31]}$, who studied whole brain NAA (WBNAA) in 43 patients with RRMS, found that no correlation between WBNAA and clinical disability using EDSS score.

\section{CONCLUSION}

Magnetic Resonance Spectroscopy is a tool that may play an important part in studies on natural history of the disease and the assessment of immunosuppressive or immunomodulatory treatment in clinical trials . It provides important data on axonal degeneration which is the subject of this study. Total T2-weighted lesion load, although more sensitive to changes with time than brain $\mathrm{N}$-acetylaspartate, may be less relevant to understanding the progression of disability.

\section{REFERENCES}

1. Compston A, Coles A : Multiple sclerosis. Lancet2008 ; 372(9648): 1502-17.

2. Streber R, Peters S, Pfeifer K : Systematic review of correlates and determinants of physical activity in persons with multiple sclerosis. Arch Phys Med Rehabil 2016

3. Milo R, Kahana $\mathbf{E}$ Multiple sclerosis: geoepidemiology, genetics and the environment. Autoimmun Rev2010; 9(5): A387-94

4. Kornek B, Lassmann H: Axonal pathology in multiple sclerosis: a historical note. Brain Pathol. 1999;(9):651-656.

5. Shailender S,Tobias D ,Anne Shanu R,Brigitte M,Heike S,Wolfgang $\mathbf{B}$,et al C: Relationship of acute axonal damage, Wallerian degeneration, and clinical disability in multiple sclerosis .Journal of Neuroinflammation 2017 ; (14):57.

6. Sorbara CD, Wagner NE, Ladwig A, Nikic I, Merkler D, Kleele T,et al :rvasive axonal transport deficits in multiple sclerosis models. Neuron.2014;(84):1183-90.

7. Haber A, LaRocca NG: Minimal Record of Disability for multiple sclerosis. New York: National Multiple Sclerosis Society; 1985

8. Ridgway JP. Cardiovascular magnetic resonance physics for clinicians: part I. J Cardiovasc Magn Reson $2010 ; 12: 71$.

9. Rosen Y, Lenkinski R. Recent advances in magnetic resonance neurospectroscopy. Neurotherapeutics 2007;4:330-45.

10.vanderGraaf $M$. In vivo magnetic resonance spectroscopy: basic methodology and clinical applications. Eur Biophys J2010 ;39:527-40. 
11.McRobbie D, Moore E, Graves M, Welcome to the MR unit. In: MRI from picture to proton. 2007;2nd edn. Cambridge University Press,:13.

12.Rooney WD, Goodkin DE, Schuff N, Meyerhoff DJ, Norman D, Weiner MW: H-MRS of normal appearing white matter in multiple sclerosis. Mult Scler. 1997, 3 (4): 231-237.

13.Leary SM, Davie CA, Parker GJ, Stevenson VL, Wang L, Barker GJ et al : $\mathrm{H}$ magnetic resonance spectroscopy of normal appearing white matter in primary progresive multiple sclerosis. J Neurol. $\quad 1999, \quad 246 \quad$ (11): 1023-1026. $10.1007 / \mathrm{s} 004150050507$.

14.Tourbah A, Stievenart JL, Gout O, Fontaine B, Liblau R, Lubetzki C: Localized proton magnetic resonance spectroscopy in relapsing remitting versus secondary progressive multiple sclerosis. Neurology. 1999, 53 (5): 1091-1097.

15. Tartaglia MC, Narayanan S, De Stefano N, Arnaoutelis R, Antel SB,et al : Choline is increased in pre lesional normal appearing white matter in multiple. J Neurol. 2002, 249 (10):

16.Enzinger C, Ropele S, Strasser-Fuchs S, Kapeller P, Schmidt H,et al : Lower levels of NAcetilaspartate in Multiple Slerosis Patients with the Apolipoprotein E 4 Allele. Arch Neurol. 2003, 60: 65-70.

17.Fernando KTM, McLean MA, Chard DT, et al. Elevated whitematter myoinositol in clinical isolated syndromes suggestive ofmultiple sclerosis. Brain 2004;127:1361-9.

18.Pan JW, Coyle PK, Bashir K, Hetherington PH. Spectroscopymetabolic differences between multiple sclerosis subtypes measured by quantitative MR. Mult Scler 2002;8:200-8.

19. Inglese M, Li BS. Diffusely elevated cerebral choline and creatine in relapsing remitting multiple sclerosis. Magn Reson Med2003;50:190-5.

20 -Brief EE, Moll R, Li DK, Mackay AL. Absolute metabolite concentrations calibrated using the total water signal in brainH1MR spectroscopy. NMR 2009;22:349-54.

21 -Kirov II, Patil V, Babb JS, Rusinek H, Herbert J. MR spectroscopy indicates diffuse multiple sclerosis activity during remission. J Neurol Neurosurg Psychiatry 2009;80:1330-6.
22-Chard D, Griffin CM, McLean MA, Kapeller P, Kapoor R, Miller DH et al: Brain Metabolites changes in cortical grey and normal appearing white matter in clinically early relapsing remitting multiple sclerosis. Brain. 2002, 125: 2342-2352.

23- Anders T,Olof D L,Jan B Marcel W,Anne A,Örjan $S$, et al :Increased Concentrations of Glutamate and Glutamine in Normal-Appearing White Matter of Patients with Multiple Sclerosis and Normal MR Imaging Brain Scans;2013.

24-Srinivasan R, Sailasuta N, Hurd R, It AL. Evidence of elevatedglutamate in multiple sclerosis using magnetic resonance spectroscopyat 3 T. Brain 2005;128:1016-625.

25-Minneboo A, Barkhof F, Polman CH, Uitdehaag $\mathrm{BM}$, Knol DL,et al :. Infra-tentorial lesions predict long-term disability in patients with initial findings suggestive of multiple sclerosis. Arch Neurol. 2004;61(2):217-221.

26--Popescu V, Agosta F, Hulst HE :Brain atrophy and lesion load predict long term disability in multiple sclerosis. J Neurol Neurosurg Psychiatry. 2013;84(10):1082-1089

27 -Adalsteinsson E, Langer-Gould A, Homer J. Gray matter Nacetylaspartate deficits in secondary progressive but not relapsingremitting multiple sclerosis. AJNR 2003;10:1941-5.

28 Rigotti DJ, Gonen O, Grossman R, Babb JS, Falini A, BenedettiB, et al. Global N-acetylaspartate declines in benign multiple sclerosis. AJNR 2011;32:204-9.

29 Wael M ,Hossam M, Doaa I, Heba A, Sabah M : Clinical use of H-MR spectroscopy in assessment of relapsing remitting and secondary progressive multiple scelorsis .The Egyptian Journal of Radiology and Nuclear Medicine.2012; 43(2);257-64.

30- Polman CH, Reingold SC, Banwell B, Clanet M, Cohen JA,et al : Diagnostic criteria for multiple sclerosis: 2010 revisions to the McDonald criteria. Ann Neurol2011; 69(2):292302

31- Kurtzke JF :Rating neurologic impairment in multiple sclerosis: an expanded disability status scale (EDSS)". Neurology 1983 ;33 (11): 144452 